\title{
QUANTIFICATION USING ULTRAVIOLET SPECTROSCOPY METHOD AND IN VITRO STABILITY STUDY OF NANOVESICULAR SYSTEM CONTAINING PHYTIC ACID
}

\author{
MALTI ARYA, KRISHNA P GUPTA, SHUBHINI A SARAF* \\ Department of Pharmaceutical Sciences, School of Biosciences and Biotechnology, Babasaheb Bhimrao Ambedkar University, Vidya Vihar, \\ Raebareli Road, Lucknow - 226 025, Uttar Pradesh, India. Email: shubhini.saraf@gmail.com
}

Received: 12 April 2018, Revised and Accepted: 08 May 2018

\begin{abstract}
Objective: The quantification of drug and stability of nanoparticulate delivery systems is one of the major apprehensions in biomedical applications. The present research work was attempted to quantify phytic acid by utilizing ultraviolet (UV) spectroscopy method and to evaluate the stability of nanovesicular (niosomes) system containing phytic acid.

Methods: Niosomes containing phytic acid were developed by thin-film hydration method. Nanoformulation was subjected to stability testing as per the International Council for Harmonisation (ICH) guidelines. The formulation was stored at $30^{\circ} \mathrm{C} \pm 2{ }^{\circ} \mathrm{C}$ and $65 \% \pm 5 \% \mathrm{RH}$, samples were withdrawn at $15^{\text {th }}, 30^{\text {th }}, 60^{\text {th }}, 90^{\text {th }}, 120^{\text {th }}$, and $180^{\text {th }}$ day of analysis and examined for the integrity of vesicular/particle size, polydispersity index, zeta potential, and percent encapsulation efficiency.
\end{abstract}

Results: Prepared nanoformulation displayed a straight line $(y=m x+c)$ equation of $y=-0.0309 x+1.0413$. Optimized batch of niosomes, which was prepared including dicetylphosphate showed zeta potential value of $-36 \pm 0.36$. Stability study showed that prepared niosomal formulation was stable up to 180 days at room temperature.

Conclusion: Findings of the current research work suggested that UV spectroscopy method can be effectively used for the quantification of phytic acid and niosomal formulation of phytic acid. The formulation was found to be stable as per the ICH guidelines for stability testing.

Keywords: Phytic acid, Niosomes, Quantification, Stability testing.

(C) 2018 The Authors. Published by Innovare Academic Sciences Pvt Ltd. This is an open access article under the CC BY license (http://creativecommons. org/licenses/by/4. 0/) DOI: http://dx.doi.org/10.22159/ajpcr.2018.v11i8.26671

\section{INTRODUCTION}

Phytic acid is a natural constituent present in almost all the cereals and legumes. It is extensively being studied to assess its effects on health and is shown to have chelation ability with minerals. It possesses various benefits on the human body such as lowering serum cholesterol, inhibitor for renal stone development, strong antioxidant, and antiangiogenic properties. It is confirmed by numerous researches that antioxidant scavenge free radicals, thus ultimately help in the cancer prevention. So far, molecular mechanism of action of phytic acid for chemoprevention is not very clear [1-3]. Previous in vitro and in vivo reports proved its anticancerous properties against prostate, colon, lung, metastatic, and mammary cancers [4].

Investigation and quantification are important elements in the formulation development of any drug. An appropriate method must exist for the quantification of the drug molecule in formulation for analysis of entrapment efficiency, dissolution studies, biological samples, etc. [5]. Ultraviolet (UV) spectroscopy is simplest and commonly used technique for such study. However, the UV region lies from 200 to $400 \mathrm{~nm}$ and visible region ranges from 400 to $800 \mathrm{~nm}$. Phytic acid does not come under the UV region. Here comes the play of colorimetric analysis. Thus, phytic acid needs chromophores to be added for its quantification through UV spectroscopy.

Nanotechnology is meant for the development of nanomedicines that deliver drugs in a sustained or controlled mode and enhances residence time of drug [6]. Nanomedicine emerged as a new epoch with growth in the application of nanotechnology in the field of diagnosis and therapy. Nanoparticles are widely being used as pharmaceutical nanocarriers, and these include metallic, polymeric, vesicular, and lipidic nanoparticles [7,8]. Non-ionic surfactant vesicles (niosomes) are of intervening importance as a delivery system $[9,10]$. Niosomes are flexible carrier which is pertinent for systemic as well as topical applications [10]. These may be uni- or multi-lamellar spheroids in structures $[11,12]$. Niosomes are formed through self-assembly of nonionic surfactant monomers which are proficient in entrapping a variety of drugs (both hydrophilic and lipophilic) [12,13]. They generally exhibit a longer storage time and may also act as targeting agents $[7,14]$.

Drug delivery systems play a key role in therapy development. The extension of the pharmaceutical industry and dosage form development is well-recognized [15]. Development of pharmaceutical products for global use necessitates that the prepared dosage form must be stable for longer duration along with persistent pharmacological potential [16]. Nanotechnologies being actively involved in the area remain linked with the development of effectual and stable dosage form [17]. To test the stability of newly developed dosage form is performed to show the evidence how properties change with the influence of time, temperature, and humidity or how long product may be used, thus to establish shelf lives $[18,19]$.

Phytic acid has momentous prospective to act as an antiangiogenic agent. Instead of having a great potential, it is grossly underutilized due to a flaw of rapid absorption and excretion from the body as it forms insoluble chelates when it comes in contact with biological ions. It is thus required to develop some novel drug delivery systems of phytic acid that can reduce its flaw, thereby increasing its anticancer effect. For the development of a novel delivery system, it is required to quantify it effectively through proper method for precise characterization. Hence, the endeavor of the current work was to quantify phytic acid and develop and characterize a niosomal delivery system of it. 


\section{METHODS}

\section{Materials}

Phytic acid and ammonium iron (III) sulfate $12 \mathrm{H}_{2} \mathrm{O}$ were purchased from Sigma Co. (St. Louis, MO). 2,2'-bipyridine was procured from Abcam. Thioglycolic acid was purchased from Merck-millipore. All other chemicals were obtained from local commercial source and were of analytical grade.

Quantitative estimation of drug through UV-spectrophotometer Quantitative estimation of phytic acid was performed as reported by Haug and Lantzsch [20]. Drug stock solution $(100 \mu \mathrm{g} / \mathrm{ml})$ was prepared in distilled water, named as the solution (i). Ferric solution was prepared separately by dissolving $0.2 \mathrm{~g}$ ammonium iron (III) sulfate. $12 \mathrm{H}_{2} \mathrm{O}$ in $100 \mathrm{ml}$ $2 \mathrm{~N} \mathrm{HCl}$ and volumes were made up to $1000 \mathrm{ml}$ with distilled water, solution named as the solution (ii). Solution (iii) was prepared by dissolving $10 \mathrm{~g}$ 2,2'-bipyridine and $10 \mathrm{ml}$ thioglycolic acid in distilled water and volume make up to $1000 \mathrm{ml}$. $0.5 \mathrm{ml}$ of drug solution was pipetted $(3-30 \mu \mathrm{g} / \mathrm{ml})$ into a test tube, $1 \mathrm{ml}$ of solution (ii) added to it and covered with parafilm. Tubes were heated in a boiling water bath for $30 \mathrm{~min}$, by taking care for the first $5 \mathrm{~min}$ that the tubes remain well covered. After that, tubes were cooled in ice water for $15 \mathrm{~min}$ and allowed to adjust to room temperature. Once the tubes had reached room temperature, the content of tubes was mixed and centrifuged for $30 \mathrm{~min}$ at $3000 \mathrm{~g} .1 \mathrm{ml}$ of the supernatant was transferred to another test tube and $1.5 \mathrm{ml}$ of solution (iii) was added. Solutions were scanned from a range of 400-600 nm, and the observed absorbance maxima value was utilized for further evaluations.

Table 1: Composition of the different batches of formulation of phytic acid loaded niosomes

\begin{tabular}{llll}
\hline Batches & $\begin{array}{l}\text { Cholesterol:surfactant } \\
\text { ratio (molar ratio) }\end{array}$ & $\begin{array}{l}\text { Sonication } \\
\text { time (min) }\end{array}$ & DCP $(\boldsymbol{\mu M})$ \\
\hline 1(NIO1) & $1: 1$ & 1 & 0 \\
2(NIO2) & $1: 2.5$ & 1 & 0 \\
3 (NIO3) & $1: 1$ & 2 & 0 \\
(NIO4) & $1: 2.5$ & 2 & 0 \\
5(NIO5) & $1: 1$ & 1 & 5 \\
6(NIO6) & $1: 2.5$ & 1 & 5 \\
7 (NIO7) & $1: 1$ & 2 & 5 \\
(NIO8) & $1: 2.5$ & 2 & 5 \\
9 (NIO9) & $1: 1.25$ & 0.5 & 2.5 \\
\hline
\end{tabular}

Table 2: Zeta potential values of phytic acid niosomes

\begin{tabular}{ll}
\hline Batches & Zeta potential \\
\hline 1 (NIO1) & $-6 \pm 0.21$ \\
(NIO2) & $-7 \pm 0.13$ \\
3(NIO3) & $-6 \pm 0.11$ \\
4 (NIO4) & $-8 \pm 0.14$ \\
5 (NIO5) & $-31 \pm 0.23$ \\
(NIO6) & $-36 \pm 0.36$ \\
(NIO7) & $-30 \pm 0.23$ \\
9(NIO) & $-29 \pm 0.31$ \\
\hline
\end{tabular}

Values are given as mean \pm SD and experiments were performed in triplicate for each batch, i.e., $\mathrm{n}=3$. SD: Standard deviation
Preparation of phytic acid containing niosomes

Niosomes were prepared through thin-film hydration method and dispersed in a suitable suspension base. Detailed preparation and characterization of the formulation are reported elsewhere [21,22]. Briefly, niosomes were prepared using Span 80-Chol-dicetylphosphate (DCP) dispersion. An appropriate quantity of cholesterol, surfactant, and stabilizing agent in variable molar ratios (Table 1) was dissolved in $10 \mathrm{ml}$ of chloroform in a round-bottomed glass flask. The chloroform was allowed to evaporate at $45^{\circ} \mathrm{C}$ under reduced pressure using a rotary evaporator (IKA ${ }^{\circledR}$ Rotavapor, Bengaluru, India). After solvent evaporation, the flask was kept overnight to remove residual solvent, resulting in the formation of a thin film. The thin film was hydrated with $5 \mathrm{ml}$ of aqueous drug solution by rotating at $45^{\circ} \mathrm{C}$ and 120 revolutions per minute (rpm). This dispersion was sonicated at $80 \%$ energy for 1 min by utilizing probe-type sonicator (Labsonic ${ }^{\circledR}$-M, Sartorius stedim) and kept for $24 \mathrm{~h}$ at $4^{\circ} \mathrm{C}$ for maturation.

\section{Zeta potential measurement}

Zeta potential of the niosomal formulation was measured using Malvern Zetasizer Nano ZS. It was determined by the electrophoretic mobility of niosome suspension in U-type tube, temperature set during the procedure was $25^{\circ} \mathrm{C}$. Zeta potential was determined through the principle of dynamic light scattering [23].

\section{Stability study of phytic acid containing niosomes}

Prepared formulation was examined for stability testing according to the International Council for Harmonisation guidelines. The rationale of stability testing is to substantiate how the properties and entrapped drug amount of formulation vary with time under the effect of temperature and humidity [24]. Optimization was done previously through various characterization parameters. The optimized batch of the niosomal suspension was used for stability testing. Stability study of the niosomal formulation was carried out by estimating the ability of the prepared system to retain the physical properties. The formulation was kept for analysis at room temperature condition, namely $30^{\circ} \mathrm{C} \pm 2^{\circ} \mathrm{C} / 60 \% \pm 5 \%$ RH. Samples were withdrawn at $15^{\text {th }}, 30^{\text {th }}$, $60^{\text {th }}, 90^{\text {th }}, 120^{\text {th }}$, and $180^{\text {th }}$ day and were examined for particle size, polydispersity index, zeta potential by Malvern Zetasizer Nano ZS, particle and zeta analyzer, and percent encapsulation efficiency through spectrophotometrically. Estimation procedure of these parameters reported elsewhere [22].

\section{RESULTS AND DISCUSSION}

Quantitative estimation of drug through UV-spectrophotometer UV-spectrophotometric method was successfully used for the quantification of phytic acid. Processed phytic acid solutions were scanned from 400 to $600 \mathrm{~nm}$, as shown in Fig. 1. Scanning showed absorbance maxima at $520 \mathrm{~nm}$, result was found in accordance with the previous report [20]. Thus, all the absorbances were measured at $520 \mathrm{~nm}$ against distilled water. A representative calibration curve was obtained which was found linear till the concentration of $30 \mu \mathrm{g} / \mathrm{ml}$. Suggested concentration range of analysis is $3-30 \mu \mathrm{g} / \mathrm{ml}$. Straight line $(y=m x+c)$ equation was found to be " $y=-0.0309 x+1.0413$ " (Fig. 2), which was utilized for all further characterizations. Negative value suggests lower the absorbance higher will be the concentration of drug present in the solution and vice versa.

Table 3: Effect of storage on particle size, PDI, zeta potential, and percent encapsulation efficiency, where n=3

\begin{tabular}{|c|c|c|c|c|}
\hline Interval in days & Particle size $(\mathrm{nm} \pm \mathrm{SD})$ & PDI (values \pm SD) & Zeta potential (values \pm SD) & Encapsulation efficiency $(\% \pm S D)$ \\
\hline 0 day & $965.5 \pm 65.2$ & $0.386 \pm 0.08$ & $-36 \pm 0.28$ & $82.9 \pm 2.6$ \\
\hline $15^{\text {th }}$ day & $966.3 \pm 71.2$ & $0.312 \pm 0.02$ & $-36 \pm 0.31$ & $81.2 \pm 3.8$ \\
\hline $30^{\text {th }}$ day & $972.3 \pm 63.2$ & $0.319 \pm 0.03$ & $-35 \pm 0.42$ & $81.3 \pm 3.6$ \\
\hline $60^{\text {th }}$ day & $979.0 \pm 76.3$ & $0.421 \pm 0.08$ & $-35 \pm 0.52$ & $80.0 \pm 4.8$ \\
\hline $90^{\text {th }}$ day & $983.5 \pm 87.4$ & $0.376 \pm 0.07$ & $-34 \pm 0.35$ & $78.3 \pm 2.8$ \\
\hline $120^{\text {th }}$ day & $988.0 \pm 75.7$ & $0.436 \pm 0.07$ & $-32 \pm 0.38$ & $78.1 \pm 4.3$ \\
\hline $180^{\text {th }}$ day & $990.3 \pm 82.3$ & $0.348 \pm 0.08$ & $-32 \pm 0.56$ & $76.0 \pm 3.6$ \\
\hline
\end{tabular}

Values have been reported as mean \pm SD, experiments were performed in triplicate for each parameter, i.e., $\mathrm{n}=3$. SD: Standard deviation, PDI: Polydispersity index 


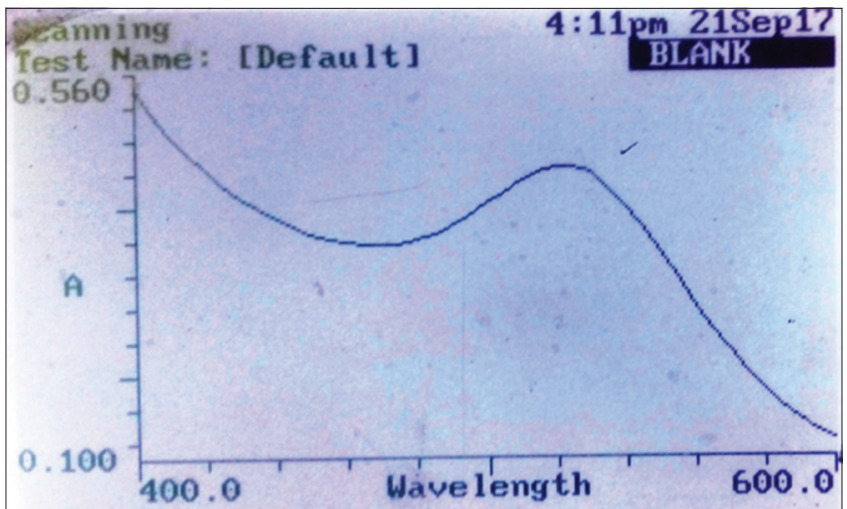

Fig. 1: Ultraviolet spectrum of phytic acid displaying it absorption maxima near $520 \mathrm{~nm}$

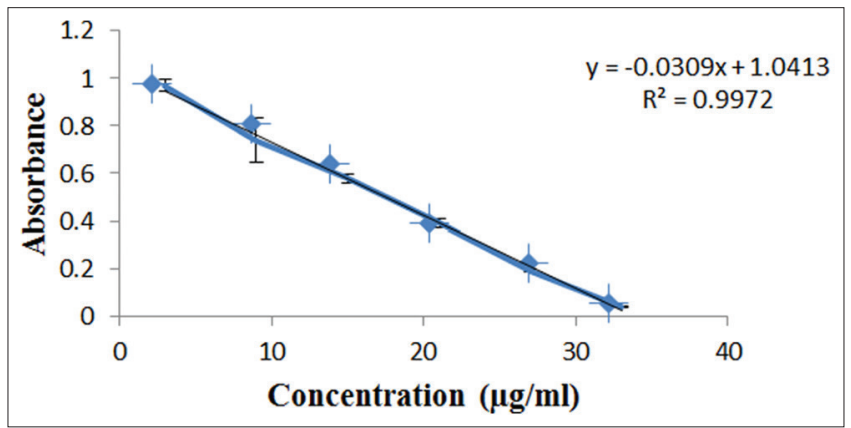

Fig. 2: Standard curve of phytic acid utilizing ultraviolet method

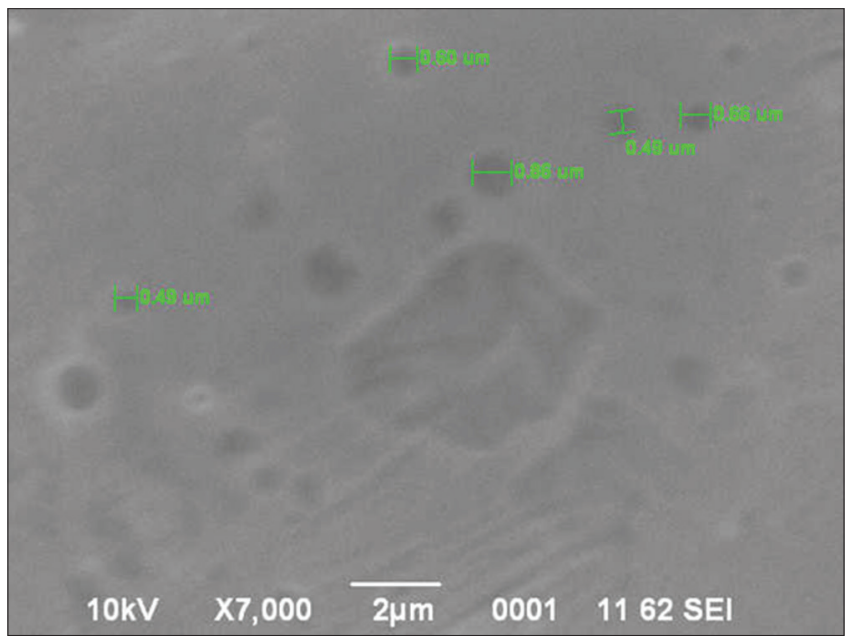

Fig. 3: Scanning electron microscope image showing spherical phytic acid niosomes

Experiments were performed in triplicate, i.e., $\mathrm{n}=3$.

Preparation and characterization of phytic acid containing niosomes

Niosomes were prepared using thin-film hydration method and were dispersed in a base which was suitable for topical application. Extensive characterization (in vitro and in vivo) of prepared niosomal formulations was performed which have been reported elsewhere [22]. Prepared niosomes, when analyzed through scanning electron microscope, were found to be spherical, as shown in Fig. 3, resembling characteristic of noisome vesicles [7].

\section{Zeta potential measurement}

Zeta potential values for prepared niosomes are presented in Table 2.
Zeta potential is related to the magnitude of the electrical charge present at the particle surface, and it indicates the degree of repulsion between adjacent particles. Either negative or positive, high zeta potential confers stability by resisting aggregation [23]. Negative value of the particle may be due to the presence of negative charge inducing agent, DCP. Batches which did not contain DCP showed lower values and those which contained DCP displayed higher values of zeta potential. This observation was in-line with characteristic property of DCP, i.e., inducing charge on the surface of the prepared vesicles to impart stability $[25,26]$.

\section{Stability study of phytic acid containing niosomes}

Optimized formulation was kept for stability testing at above-mentioned condition. Results of stability study showed that the prepared niosomal formulation was stable up to 180 days at room temperature (Table 3). All the parameters were found stable during the analysis. This may be attributed to the presence of DCP which has the property to induce charge on the surface of the vesicles and would not allow its aggregation, thereby enhancing its stability $[25,26]$. No significant degradation was observed on storage at the condition of $30^{\circ} \mathrm{C} \pm 2^{\circ} \mathrm{C} / 60 \% \pm 5 \%$. Thus, shelf life of prepared formulation is estimated to be high.

\section{CONCLUSION}

In the present study, the findings revealed that UV spectroscopy method can be efficiently utilized for the quantification of phytic acid. DCP may be utilized as a charge inducing and stabilizing agent for the preparation of niosomes. Niosomal formulation of phytic acid was successfully prepared using DCP and through thin-film hydration method. Thus, prepared system was stable up to 180 days, i.e., it can be stored at room temperature for sufficient period.

\section{AUTHOR'S CONTRIBUTION}

M.A. performed all the bench work. K.P.G. and S.A.S. helped in conceptualizing and overall guidance of the work.

\section{CONFLICTS OF INTEREST}

All authors have no conflicts of interest to declare.

\section{REFERENCES}

1. Chahal AK, Chhillar AK, Saini RV. Natural antioxidants as defense system against cancer. Asian J Pharm Clin Res 2018;11:38-44.

2. Gupta KP, Singh J, Bharathi R. Suppression of DMBA-induced mouse skin tumor development by inositol hexaphosphate and its mode of action. Nutr Cancer 2003;46:66-72.

3. Pandey M, Gupta KP. Epigenetics, an early event in the modulation of gene expression by inositol hexaphosphate in ethylnitrosourea exposed mouse lungs. Nutr Cancer 2011;63:89-99.

4. Saad N, Esa NM, Ithnin H, Shafie NH. Optimization of optimum condition for phytic acid extraction from rice bran. Afr J Plant Sci 2011;5:168-75.

5. Patel J, Kevin G, Patel A, Raval M, Sheth N. Development of the UV spectrophotometric method of Olmesartan medoxomil in bulk drug and pharmaceutical formulation and stress degradation studies. Pharm Methods 2011;2:36-41

6. Hemant KS, Raizaday A, Sivadasu P, Uniyal S, Kumar SH. Cancer nanotechnology: Nanoparticulate drug delivery for the treatement of cancer. Int J Pharm Pharm Sci 2014;7:40-6.

7. Moghassemi S, Hadjizadeh A. Nano-niosomes as nanoscale drug delivery systems: An illustrated review. J Control Release 2014; 185:22-36.

8. Karna SC, Agrawal V, Alim M. Formulation approaches for sustained release dosage forms: A review. Asian J Pharm Clin Res 2015;8:46-53.

9. Escudero I, Geanta RM, Ruiz MO, Benito JM. Formulation and characterization of Tween 80/cholestherol niosomes modified with tri-n-octylmethylammonium chloride (TOMAC) for carboxylic acids entrapment. Colloids Surf A Physicochem Eng Asp 2014;461:167-77.

10. Paolino D, Cosco D, Muzzalupo R, Trapasso E, Picci N, Fresta M. Innovative bola-surfactant niosomes as topical delivery systems of 5-fluorouracil for the treatment of skin cancer. Int $\mathrm{J}$ Pharm 2008;353:233-42. 
11. Balakrishnan P, Shanmugam S, Lee WS, Lee WM, Kim JO, Oh DH, et al. Formulation and in vitro assessment of minoxidil niosomes for enhanced skin delivery. Int J Pharm 2009;377:1-8.

12. Ruckmani K, Sankar V. Formulation and optimization of zidovudine niosomes. AAPS Pharmscitech 2010;11:1119-27.

13. Surya TS, Mothilal M, Damodharan N, Jaison D. Screening and optimization of valacyclovir niosomes by design of experiments. Int $\mathrm{J}$ App Pharm 2018;10:79-85

14. Okore V, Attama A, Ofokansi K, Esimone C, Onuigbo E. Formulation and evaluation of niosomes. Indian J Pharm Sci 2011;73:323.

15. Asthana GS, Sharma $\mathrm{PK}$, Asthana A. In vitro and in vivo evaluation of niosomal formulation for controlled delivery of clarithromycin. Scientifica 2016;2016:.

16. Mathew B, Rangapriya M, Rajendran N. Evaluation and stability studies of rifampicin loaded chitosan nanoparticle. World J Pharm Pharm Sci 2014;3:536-57.

17. Zamboni WC, Torchilin V, Patri AK, Hrkach J, Stern S, Lee R, et al. Best practices in cancer nanotechnology: Perspective from NCI nanotechnology alliance. Clin Cancer Res 2012;18:3229-41.

18. Rezayat M. Preparation, characterization and stability investigation of chitosan nanoparticles loaded with the Echis carinatus snake venom as a novel delivery system. Arch Razi Institute 2015;70:269-77.

19. Ertekin ZC, Bayindir ZS, Yuksel N. Stability studies on piroxicam encapsulated niosomes. Curr Drug Deliv 2015;12:192-9.

20. Haug W, Lantzsch HJ. Sensitive method for the rapid determination of phytate in cereals and cereal products. J Sci Food Agric 1983;34:1423-6.

21. Acharya A, Kumar GB, Ahmed MG, Paudel S. Niosome-encapsulated clomipramine for transdermal controlled delivery. Int J Pharm Pharm Sci 2014;6:567-75

22. Arya M, Tiwari P, Tripathi CB, Parashar P, Singh M, Sinha P, et al. Colloidal vesicular system of Inositol hexaphosphate to counteract DMBA induced dysregulation of markers pertaining to cellular proliferation/differentiation and inflammation of epidermal layer in mouse model. Mol Pharm 2017;14:928-39.

23. Kanoujia J, Singh M, Singh P, Parashar P, Tripathi CB, Arya M, et al. Genipin crosslinked soy-whey based bioactive material for atorvastatin loaded nanoparticles: Preparation, characterization and in vivo antihyperlipidemic study. RSC Adv 2016;6:93275-87.

24. Branch SK. Guidelines from the international conference on harmonisation (ICH). J Pharm Biomed Anal 2005;38:798-805.

25. Abdelbary G, El-gendy N. Niosome-encapsulated gentamicin for ophthalmic controlled delivery. AAPS Pharmscitech 2008;9:740-7.

26. Bendas ER,Abdullah H,El-Komy MH, Kassem MA. Hydroxychloroquine niosomes: A new trend in topical management of oral lichen planus. Int $\mathrm{J}$ Pharm 2013;458:287-95. 